\title{
A case control and family based association study of the neuregulin 1 gene and schizophrenia
}

\author{
$X$ Zhao, Y Shi, J Tang, R Tang, L Yu, N Gu, G Feng, S Zhu, H Liu, Y Xing, S Zhao, H Sang, Y Guan, \\ D St Clair, L He
}

J Med Genet 2004;41:31-34

D ata from twin, family, and adoption studies provide strong evidence that genetic factors play a major aetiological role in schizophrenia. By a series of linkage studies, chromosome $8 \mathrm{p}$ has been implicated as a region harbouring a schizophrenia susceptibility gene. ${ }^{1-4}$ Recently, Stefansson and colleagues reported that neuregulin 1 (NRG1), located in 8p21-12, may be involved in the aetiology of schizophrenia. ${ }^{45}$ In their linkage and association studies, a $290 \mathrm{~kb}$ core at risk haplotype at the $5^{\prime}$ end of NRGI was found to be strongly associated with schizophrenia in Icelandic and Scottish populations. This haplotype contains the first exon of NRG1, which encodes a part of glial growth factor 2 (GGF2). Deficiency of glial growth factors has been presumed to be implicated in the pathogenesis of schizophrenia. ${ }^{6}$ Futhermore, NRGI mutant mice have fewer functional $\mathrm{N}$-methyl D-aspartate(NMDA) receptors than wild type mice, and display stereotypic behavioural abnormalities similar to those of normal mice treated with the psychogenic drug phenylcyclidine. $^{4}$

This core at risk haplotype was defined by five single nucleotide polymorphisms (SNP8NRG221132, SNP8NRG221533, SNP8NRG241930， SNP8NRG243177， SNP8NRG433E1006) and two microsatellites (478B14-848, 420M91395). The frequency of this haplotype in schizophrenic individuals was higher than in controls; in Icelandic samples the frequency was $15.4(7.5 \%$; $=0.000087) .{ }^{4}$ The first replication using Scottish samples revealed a similar result at 10.2 (5.9\%; $\mathrm{p}=0.00031) .{ }^{5}$ Another replication performed by Williams et al with British or Irish samples used one SNP and the two microsatellites of the core at risk haplotype. However, the association was much weaker at $9.5(7.5 \%$; $\mathrm{p}=0.04){ }^{7}$ Yang et al reported other markers located in the middle of NRG1 and associated with schizophrenia, in a Chinese population. ${ }^{8}$ Another independent analysis using 13 microsatellites found two groups of haplotypes, which were significantly associated with schizophrenia, in a Chinese population. ${ }^{9}$

All these studies suggest that NRG1 may be a susceptibility gene for schizophrenia. However, there are differences in the findings and more replications are needed. Here we present results from the haplotype analysis using both case control and TDT. In our studies, only three of the five SNPs (SNP8NRG221533， SNP8NRG241930, SNP8NRG243177) and the two microsatellites reported by Stefansson et al were genotyped. The marker SNP8NRG221132 was abandoned because of low heterozygosity. We genotyped more than 200 individuals at this locus, but no allele A was found. In all, 1724 Han people Chinese participated in our research, including 369 subjects with schizophrenia, 299 controls in the case control study, and 352 family trios in the TDT study.

\section{MATERIALS AND METHODS}

For the case control investigation, 369 unrelated persons with schizophrenia (178 were male and 191 were female, with a

\section{Key points}

- In a sample of 1724 Han Chinese individuals, 3 ummSNPs (single nucleotide polymorphisms) and 2 microsatellites were genotyped. Case control and TDT analyses were performed to investigate whether the $5^{\prime}$ end of neuregulin 1 (NRG1) was associated with schizophrenia.

- Owing to the low frequency found of the reported at risk haplotype $(<1 \%)$, no association could be detected with schizophrenia. However, another haplotype was found to be significantly associated with schizophrenia in both case control ( $p=0.0057)$ and TDT analyses ( $p=0.0043)$.

- The results support the view that NRG1 may be an important factor in the aetiology of schizophrenia.

mean age of 41.56 years, $S D=14.35)$ and 299 control individuals ( 148 were male and 151 were female, with a mean age of 31.26 years, SD $=9.07$ ) were recruited. The cases included 177 patients from Shanghai and 192 patients from JiLin; the controls comprised 109 people from Shanghai and 190 people from JiLin.

For the TDT study, 352 unrelated schizophrenia probands ( 187 were male and 165 were female, with a mean age of 23.86 years, $\mathrm{SD}=6.60$ ) and their biological parents were recruited. All subjects were Han Chinese in origin. Those with schizophrenia were diagnosed strictly according to the criteria of DSM-III-R (American Psychiatric Association, 1987). Written informed consent was obtained from either the participants or the participants' relatives, after the procedure had been fully explained.

High molecular weight genomic DNA was prepared from venous blood using the standard phenol chloroform extraction. SNPs were genotyped through TaqMan ${ }^{\circledR}$ technology on an ABI7900 system and probes and primers were designed by the Assay-by-Design ${ }^{\circledR}$ service of Applied Biosystems (San Jose, CA, USA). The standard PCR reactions of $5 \mu \mathrm{l}$ were carried out using TaqMan ${ }^{\circledR}$ Universal PCR Master Mix reagent kits as in the guidelines. During assay development, microsatellite 478B14-848 was amplified with upper primer 5' -cca cat gtc caa ctg aag agg- 3' and lower primer 5' - cag aga cat cct gta tga cca aa- 3'; microsatellite 420M9-1395 was amplified with upper primer $5^{\prime}$ - tgt tgt tgt ata tt cag aat ttc ctt- 3' and lower primer $5^{\prime}$-att cca gtt aaa aga tga aaa gag c- 3'. Each upper primer was fluorescently labelled. PCR products were electrophoresed on MegaBACE 1000 instruments (Amersham Biosciences, Amersham, UK). In order to compare the results, we aligned our allele histograms with allele histograms for a Chinese population genotyped at deCODE. 
Table 1 Allele frequencies of all markers and association analysis of individual markers

\begin{tabular}{|c|c|c|c|c|c|c|c|c|}
\hline \multirow[b]{2}{*}{ Marker* } & \multirow[b]{2}{*}{ Allele } & \multicolumn{3}{|c|}{ Case control study } & \multicolumn{4}{|l|}{ TDT study } \\
\hline & & Caset & Controlt & $p$ & Probandst & Parents† & T:NT & $\mathbf{p}$ \\
\hline SNP8NRG221533 & C & $396(0.54)$ & $263(0.52)$ & 0.56 & $385(0.57)$ & $742(0.55)$ & 173:149 & 0.18 \\
\hline SNP8NRG241930 & G & $681(0.93)$ & $560(0.95)$ & 0.14 & $658(0.95)$ & $1293(0.94)$ & $47: 30$ & 0.053 \\
\hline SNP8NRG243177 & $\mathrm{T}$ & $396(0.57)$ & $325(0.56)$ & 0.91 & $403(0.60)$ & $796(0.59)$ & $155: 143$ & 0.49 \\
\hline $478 \mathrm{~B} 14-848$ & 4 & $427(0.58)$ & $296(0.54)$ & $0.15 \ddagger$ & $397(0.58)$ & $722(0.58)$ & $166: 141$ & $0.17 \ddagger$ \\
\hline 420M9-1395 & -2 & $498(0.68)$ & $402(0.67)$ & $0.17 \ddagger$ & $456(0.66)$ & $796(0.65)$ & $139: 111$ & $0.18 \ddagger$ \\
\hline
\end{tabular}

*Marker names used by Stefansson et al, 2002. †Allele frequencies are shown in parentheses. $¥$ Global $p$ values of the $\chi^{2}$ statistics.

$p$, $p$ value; $T$, transmissions from heterozygous parent to affected offspring; NT, non-transmissions.

For case control analysis, the statistical significance of differences in the allele and haplotype frequency distributions between patients and controls was estimated using the program Clump 2.2. ${ }^{10}$ The software was also used to compare genotype frequencies between Shanghai samples and JiLin samples. Because no differences were found, samples from the different regions were grouped together in later analyses. Each computation was performed with at least 100000 simulations. The multiple markers' haplotype frequencies were estimated using the program EHPLUS. ${ }^{11}{ }^{12}$

For TDT analysis, ETDT ${ }^{13}$ was used to perform the transmission disequilibrium test for single markers. Haplotypic analysis was carried out by TRAMSMIT, ${ }^{14}$ version 2.5.4.

The standardised measure of LD for each pair of markers, denoted as $\mathrm{D}^{\prime}$, was estimated with software $2 \mathrm{LD}^{15}$ for both case control and TDT analysis. All tests were two tailed and significance was accepted at $\mathrm{p}<0.05$. To allow comparisons with previous studies, significance levels were not adjusted for multiple testing.

\section{RESULTS}

All the markers showed obvious differences in allele frequencies in our Chinese population, compared with Stefansson's results. ${ }^{45}$ Allele frequencies and single marker analyses are shown in table 1 . None of the five markers revealed significant allelic association in case control samples nor transmission distortion in trios.

We calculated linkage disequilibrium (LD) for all marker pairs (expressed in $\mathrm{D}^{\prime}$ ). Strong LD was observed in all SNP pairs and microsatellite pairs $\left(\mathrm{D}^{\prime}>0.6\right)$, whereas LD between SNPs and microsatellites was much weaker $\left(\mathrm{D}^{\prime}<0.4\right)$. The results were consistent in both case control samples and family trios. They also accorded with the results of Tang et al, who suggested that there may be a recombination breakpoint between the two microsatellites 478B14-642 (between SNP8NRG221533 and SNP8NRG241930) and 487-2 (upstream of 478B14-848). ${ }^{9}$

Results of haplotype analysis are shown in tables 2 and 3: 15 common haplotypes with probability $>1 \%$ were present in $86 \%$ of cases, $87 \%$ of controls, and $83 \%$ of trios. Global $\chi^{2}$ test of haplotypes in case control samples showed significant association with schizophrenia $\left(\chi^{2}=34.36\right.$; $15 \mathrm{df} ; \mathrm{p}=$ $0.0035)$. In TDT analysis, we obtained similar results, although the association was weaker $\left(\chi^{2}=28.17 ; 15 \mathrm{df}\right.$; $\mathrm{p}=0.020)$.

The frequency of the risk haplotype identified by Stefansson was less than $1 \%$ in the Chinese population. Another haplotype, $\mathrm{C} / \mathrm{G} / \mathrm{T} / 4 /-2$, showed an excess in the case control study (37.1\%: $27.5 \%$; $\mathrm{p}=0.0057$; OR $=1.56$ ). This haplotype was also over transmitted in trios $(\mathrm{p}=$ 0.0046).

We analysed the SNPs and the microsatellites as two blocks, to compare our results with previous studies. No significant results were obtained in the analysis of the three SNP haplotypes. However, the haplotypes that consisted of the two microsatellites showed an association with schizophrenia in case controls $(p=0.0018)$ and in TDT analysis of trios ( $p=0.038$ ); the haplotype $4 /-2$ contributed most to the association $(p=0.0044$ for case control samples and $\mathrm{p}=0.018$ for trios). Our data on the haplotypes consisting of two microsatellites confirmed the association of a block of microsatellites (487-2, 478B14-848, 420M9-1395, D8S1810, 420M9-3663) reported by Tang et al. In their study, the haplotype specified as 20/4/-2/18/0 (487-2, 478B14-848, 420M9-1395, D8S1810, 420M9-3663) generated the positive association, and in our study the corresponding haplotype with alleles 4/-2 (478B14-848, 420M9-1395) played the same role. The relation between the current and previous studies is shown in table 4.

\section{DISCUSSION}

In the current study, we replicate the association between the $5^{\prime}$ end of NRG1 and schizophrenia in a Chinese population. Although the risk haplotype differs from that of Stefansson et al, which was virtually absent in the Chinese population, our results provide important evidence to support the existence of one or more functional variants within this region in both populations. As the given region covers the first promoter of NRG1 and the first exon of GGF2, the

Table 2 Case control analysis of the risk haplotype $\mathrm{C} / \mathrm{G} / \mathrm{T} /-4 / 2$

\begin{tabular}{|c|c|c|c|c|}
\hline Haplotype & $\begin{array}{l}\text { Frequency* } \\
\text { (cases) }\end{array}$ & $\begin{array}{l}\text { Frequency* } \\
\text { (controls) }\end{array}$ & p Valuet & Odds ratiosł \\
\hline $\begin{array}{l}5 \text { markers } \\
\text { haplotype }\end{array}$ & 37.1 & 27.5 & $\begin{array}{c}0.0057 \\
(0.00069)\end{array}$ & $\begin{array}{l}1.56 \\
(1.20-2.01)\end{array}$ \\
\hline $\begin{array}{l}3 \text { SNPs } \\
\text { haplotype(CGT) }\end{array}$ & 51.1 & 50.3 & $\begin{array}{l}0.91 \\
(0.90)\end{array}$ & $\begin{array}{l}1.02 \\
(0.81-1.28)\end{array}$ \\
\hline $\begin{array}{l}\text { Microsatellites } \\
\text { haplotype (4/-2) }\end{array}$ & 57.2 & 48.9 & $\begin{array}{c}0.0061 \\
(0.00078)\end{array}$ & $\begin{array}{l}1.40 \\
(1.13-1.72)\end{array}$ \\
\hline
\end{tabular}

*Frequency \%. †Values in parentheses are uncorrected $p$ values from $\chi^{2}$ distribution.

$\ddagger$ Values in parentheses are $95 \%$ confidence intervals.

Table 3 Transmission disequilibrium test of the risk haplotype C/G/T/-4/2

\begin{tabular}{llll}
\hline Haplotype & $\begin{array}{l}\text { Observed } \\
\text { result }\end{array}$ & $\begin{array}{l}\text { Expected } \\
\text { result }\end{array}$ & p Value \\
\hline 5 markers haplotype & 249.19 & 225.25 & 0.0046 \\
3SNPs haplotype(CGT) & 375.80 & 358.86 & 0.060 \\
$\begin{array}{l}\text { Microsatellites } \\
\text { haplotype(4/-2) }\end{array}$ & 380.75 & 359.11 & 0.018 \\
\hline
\end{tabular}




\begin{tabular}{|c|c|c|c|c|}
\hline \multirow[b]{2}{*}{ Marker } & \multirow[b]{2}{*}{ Interval(kb) } & \multicolumn{3}{|c|}{ Comparison of current and previous studies } \\
\hline & & Stefansson $^{45}$ & Tang' & Our study \\
\hline SNP8NRG221132 & - & * & - & - \\
\hline SNP8NRG221533 & 0.4 & * & - & * \\
\hline $478 B 14-642$ & 18.7 & - & - & - \\
\hline SNP8NRG241930 & 1.3 & * & - & * \\
\hline SNP8NRG243177 & 1.25 & * & - & * \\
\hline SNP8NRG433E1006 & 2.1 & * & - & - \\
\hline $487-2$ & 78.0 & - & * & - \\
\hline 478B14-848 & 20.0 & * & * & * \\
\hline 420M9-1395 & 77.0 & * & * & * \\
\hline D8S1810 & 26.0 & - & * & - \\
\hline $420 \mathrm{M} 9-3663$ & 10.0 & - & - & - \\
\hline Risk haplotype $\dagger$ & - & $\mathrm{G} / \mathrm{C} / \mathrm{G} / \mathrm{T} / \mathrm{G} / 0 / 0$ & $20 / 4 /-2 / 18 / 0$ & $\mathrm{C} / \mathrm{G} / \mathrm{T} / \mathbf{4} /-2$ \\
\hline
\end{tabular}

variant may affect the expression or splicing of the GGF2 isoform. ${ }^{6}$

These differences in allele frequencies, LD, and haplotype indicate that there may be more than one functional variant in the region, spanning several hundred kilobases. Alternatively, the same functional variant, which has not been identified, may be carried on a different haplotype in the Chinese population $v$ Caucasians.

The results of TDT in trios, when compared with the case control study, show a much weaker association. There are two main explanations that could account for our results. First, case control study has more statistical power than TDT to detect associations, because only parents who are heterozygous in given loci provide effective information in TDT. ${ }^{16}$ In the present study, heterozygosity of the microsatellites was limited, and the two haplotypes with the highest frequencies accounted for more than $70 \%$ of the total; thus the results of case control differed considerably from those of TDT. Secondly, it is possible that part of positive association is contributed by stratification, and TDT may avoid this problem effectively. ${ }^{17}$ However, the association detected by our case control study should not be considered a false positive, seeing that significant association was obtained by TDT as well.

The $\mathrm{p}$ values of risk haplotypes are also distorted by these factors. However, in our case control study, p values were assessed using the Monte Carlo approach instead of $\chi^{2}$ distribution, so that the $\mathrm{p}$ values have been corrected. ${ }^{10}$ Corrected and uncorrected p values are shown in table 2; the $\mathrm{p}$ values of TDT are not corrected.

In conclusion, our results confirm some previous studies and provide further support for the importance of NRGI in predisposition to schizophrenia. However, the functional variant harbouring in this region is still unknown, and further detailed LD mapping in different populations is essential.

\section{ACKNOWLEDGEMENTS}

This work was supported by grants from the National 863 and 973 Projects, the National Natural Science Foundation of China, the Shanghai Municipal Commission for Science and Technology, and the Qiu Shi Science \& Technologies Foundation.

\section{Authors' affiliations}

X Zhao, Y Shi, J Tang, R Tang, L Yu, L He, Bio-X Life Science Research Center, Shanghai Jiao Tong University, Shanghai, China

$X$ Zhao, Y Shi, J Tang, R Tang, L Yu, L He, Institute of Nutrition Science, Shanghai Institutes for Biological Sciences, Chinese Academy of Sciences, Shanghai, China
N Gu, G Feng, Shanghai Institute of Mental Health, Shanghai, China S Zhu, H Liu, Jilin Institute of Mental Health, Jilin, China

Y Xing, S Zhao, Xi'an Institute of Mental Health, Xi'an, China

H Sang, Y Guan, Changchun Kaixuan Hospital, Changchun, China

D St Clair, Department of Mental Health, University of Aberdeen, Aberdeen, UK

$X$ Zhao and $Y$ Shi have contributed equally to this work.

Correspondence to: L He, Shanghai Jiao Tong University, Bio-X Life Science Research Center, PO Box 501, Hao Ran Building, 1954 Hua Shan Road, Shanghai 200030, China; helin@nhgg.org

Received 26 September 2003

Accepted 26 October 2003

\section{REFERENCES}

1 Blouin JL, Dombroski BA, Nath SK, Lasseter VK, Wolyniec PS, Nestadt G Thornquist M, Ullrich G, McGrath J, Kasch L, Lamacz M, Thomas MG Gehrig C, Radhakrishna U, Snyder SE, Balk KG, Neufeld K, Swartz KL, DeMarchi N, Papadimitriou GN, Dikeos DG, Stefanis CN, Chakravarti A, Childs B, Pulver AE. Schizophrenia susceptibility loci on chromosomes 13q32 and 8p21. Nat Genet 1998;20:70-3.

2 Gurling HM, Kalsi G, Brynjolfson J, Sigmundsson T, Sherrington R, Mankoo BS, Read T, Murphy P, Blaveri E, McQuillin A, Petursson H, Curtis D. Genomewide genetic linkage analysis confirms the presence of susceptibility loci for schizophrenia, on chromosomes 1q32.2, 5q33.2, and 8p21-22 and provides support for linkage to schizophrenia, on chromosomes 1 lq23.3-24 and 20q12.1-11.23. Am J Hum Genet 2001;68:661-73.

3 Kendler KS, Myers JM, O'Neill FA, Martin R, Murphy B, MacLean CJ, Walsh D, Straub RE. Clinical features of schizophrenia and linkage to chromosomes 5q, 6p, 8p, and 10p in the Irish Study of High-Density Schizophrenia Families. Am J Psychiatry 2000;157:402-8.

4 Stefansson $\mathrm{H}$, Sigurdsson E, Steinthorsdottir V, Biornsdottir S, Sigmundsson T, Ghosh S, Brynjolfsson J, Gunnarsdottir S, Ivarsson O, Chou T, Hjaltason O, Birgisdottir B, Jonsson H, Gudnadottir VG, Gudmundsdottir E, Bjornsson A, Ingvarsson $B$, Ingason A, Sigfusson S, Hardardottir $H$, Harvey RP, Lai D, Zhou M, Brunner D, Mutel V, Gonzalo A, Lemke G, Sainz J, Johannesson G, Andresson T, Gudbjartsson D, Manolescu A, Frigge ML, Gurney ME, Kong A, Gulcher JR, Petursson H, Stefansson K. Neuregulin 1 and susceptibility to schizophrenia. Am J Hum Genet 2002;71:877-92.

5 Stefansson H, Sarginson J, Kong A, Yates P, Steinthorsdottir V, Gudfinnsson E, Gunnarsdottir S, Walker N, Petursson H, Crombie C, Ingason A, Gulcher JR, Stefansson K, St Clair D. Association of neuregulin 1 with schizophrenia confirmed in a Scottish population. Am J Hum Genet 2002;72:83-7.

6 Moises HW, Zoega T, Gottesman II. The glial growth factors deficiency and synaptic destabilization hypothesis of schizophrenia. BMC Psychiatry 2002;2:8.

7 Williams NM, Preece A, Spurlock G, Norton N, Williams HJ, Zammit S, O'Donovan MC, Owen MJ. Support for genetic variation in neuregulin 1 and susceptibility to schizophrenia. Mol Psychiatry 2003;8:485-7.

8 Yang JZ, Si TM, Ruan Y, Ling YS, Han YH, Wang XL, Zhou M, Zhang HY, Kong QM, Liu C, Zhang DR, Yu YQ, Liu SZ, Ju GZ, Shu L, Ma DL, Zhang D. Association study of neuregulin 1 gene with schizophrenia. Mol Psychiatry 2003;8:706-9

9 Tang JX, Chen WY, He G, Zhou J, Gu NF, Feng GY, He L. Polymorphisms within $5^{\prime}$ end of the neuregulin 1 gene are genetically associated with schizophrenia in the Chinese population. Mol Psychiatry (in press). 
10 Sham PC, Curtis D. Monte Carlo tests for associations between disease and alleles at highly polymorphic loci. Ann Hum Genet 1995;59:97-105.

11 Zhao J, Curtis D, Sham PC. Model-free analysis and permutation tests for allelic associations. Hum Hered 2000;50:133-9.

$12 \mathrm{Xie} \mathbf{X}, \mathrm{Ott} J$. Testing linkage disequilibrium between a disease gene and marker loci. Am J Hum Genet 1993;53:1107.

13 Sham PC, Curtis D. An extended transmission/disequilibrium test (TDT) for multi-allele marker loci. Ann Hum Genet 1995:59:323-66.
14 Clayton D. A generalization of the transmission/disequilibrium test for uncertain-haplotype transmission. Am J Hum Genet 1999;65:1170-7.

15 Zapata C, Carollo C, Rodriguez S. Sampling variance and distribution of the $D^{\prime}$ measure of overall gametic disequilibrium between multiallelic loci. Ann Hum Genet 2001;65:395-406.

16 Jorde LB. Linkage disequilibrium and the search for complex disease genes. Genome Res 2000;10:1435-44.

17 Ewens WJ, Spielman RS. The transmission/disequilibrium test: history, subdivision, and admixture. Am J Hum Genet 1995;57:455-64.

\section{$\mathrm{ECHO}$}

\section{BRAF mutations in cholangiocarcinoma}

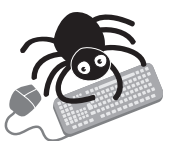

Please visit the Journal of Medical Genetics website [www. imedgenet. com] for a link to the full text of this article. ntrahepatic cholangiocarcinoma is the second most common primary hepatic malignant neoplasm, after hepatocellular carcinoma. Retrovirus-associated DNA sequences (RAS), controlled by RAS oncogenes, works at least in part through the mitogen activated protein kinase (MAPK) signal transduction cascade. Signalling through this cascade leads to activation of RAF kinase. Mammalian cells contain three RAF isoforms, A-RAF, B-RAF, and C-RAF. Mutations of BRAF have been found in around $15 \%$ of all human cancers, especially in malignant melanomas. Now researchers in Leipzig have studied the role of BRAF in liver tumours.

They looked for BRAF and KRAS mutations in 25 hepatocellular carcinomas and 69 cholangiocarcinomas by direct DNA sequencing after microdissection. MAPK pathway active intermediates were detected using immunohistochemistry. Activating BRAF missense mutations were found in 15 cholangiocarcinomas (22\%) and in none of the hepatocellular carcinomas. The mutations were not found in non-neoplastic liver tissue. All BRAF mutations were within exons 11 and 15 and 11 of 15 mutations were in nucleotide 1796 leading to substitution of valine by glutamic acid at position 599.

KRAS mutations were found in 31 cholangiocarcinomas $(45 \%)$ and none of the hepatocellular carcinomas. Twenty four mutations were of codon 12 and seven of codon 11. Ten were $\mathrm{G} \rightarrow \mathrm{A}$ transitions. Two KRAS mutations were found in non-neoplastic tissue. No correlations were observed between BRAF or KRAS mutations and histological or clinical features. Disruption of the Raf/MEK/ERK (MAPK) kinase pathway by either KRAS or BRAF mutation was detected in approximately $62 \%$ of all cholangiocarcinomas.

$B R A F$ and KRAS mutations were common in cholangiocarcinoma but were not found in hepatocellular carcinoma.

\ Gut 2003;52:706-12. 